\title{
A CLOSED-LOOP CONTROLLED EQUIPMENT FOR SOIL CONSOLIDATION TESTS
}

\author{
G. Conte* - S. Mignozzetti* - G. Scarpelli** - F. Tenti** \\ * Dept. Electronics and Automation - **Dept. Material and Earth Science \\ University of Ancona - via Brecce Bianche 60131 Ancona - Italy \\ gconte(opopcsi.umian .it-g.scarpelliajgeotecnica.unian.it
}

\begin{abstract}
A novel equipment for consolidation tests of soil samples is described. The equipment consists of a press and an oedometric cell equipped with a closed-loop control architecture. Closed-loop control is used for regulating the compression process of a soil sample according to the dynamical behaviour of the interstitial pressure, in such a way to emulate natural conditions. The equipment allows to perform consolidation tests of a new kind and to collect data for deducing Consolidation Curves with accuracy and, in comparison with clasșic methodologies, in a very short time.
\end{abstract}

Keywords: closed-loop control, soil consolidation tests

\section{INTRODUCTION}

In order to guarantee stability and serviceability of buildings and other constructions, it is of crucial importance to know the behaviour of the soil in response to the actions exerted by the foundations. Relevant behavioural parameters are currently estimated by carrying on specific consolidation tests in which, essentially, soil samples are compressed and the pore water they contain is drained. The necessity, during these laboratory tests, of emulating the conditions under which the consolidation process occurs in nature imposes to keep the value of specific variables, like for instance internal and external pressures or other forces, within fixed bounds. Using traditional laboratory equipment, this is generally achieved by controlling in an open-loop mode the behaviour of those variables. The efficacy of openloop control is practically guaranteed by exploiting the natural damping characteristics of the processes at issue, at the price of increasing, over several days, the duration of the tests. Obviously, this has also the undesirable effect of increasing the cost of the tests. A possible way to overcome these drawbacks consists in developing laboratory equipment which basically, thank to suitable control architecture, operate automatically under closed-loop control (compare with [2]).

The principal advantage that is obtained in this way consists in a substantial reduction of the duration of laboratory tests, without renouncing to emulate carefully natural conditions. Consequently, costs are reduced and reliability, reproducibility and casiness are increased. In addition, two other positive effects deserve to be pointed out. The first one is that the data used for implementing closed-loop control represent the behaviour of specific variables at a high sampling rate and, therefore, they may provide a more accurate description of the processes at issue than that obtained by a sequence of manually collected measures. The second one is that the use of reliable automatic control procedures on laboratory equipment can facilitate its integration under a supervisory unit and to reduce human intervention in routine as well as in complex tests. The above motivations are at the base of the work done in recent years by the authors in designing automatic laboratory equipment for consolidation tests.

In the paper, a novel equipment for consolidation tests of fine grained soil samples is described. The equipment consists of a press and an oedometric cell equipped with a closed-loop control architecture. Closed-loop control is used for regulating the compression process of the soil sample according to the dynamical behaviour of the interstitial pressure, in such a way to meet the required conditions for consolidation testing. The actuated control strategy is hybrid, in order to comply with different requirements in the pre-compression phase and in the compression phase of the process. During the compression phase the control signal is generated by a PID controller implemented by means of a PC, 
equipped with a friendly man/machine interface.

The equipment can be used for performing innovative soil compression tests. which by comparison with more common geotechnical laboratory procedures. are shown to provide reliable results for deriving soil parameters useful in design.

Widh respect to classical tests. however. the time necessary for acquiring data is largely reduced (up to six times in average situations). These results may potentially promote a large use of innovative equipment and testing procedures of the kind described in the paper as well as foster the role of automation in the development of advanced laboratory equipment for geoteclunical tests.

\section{THE CLOSED-LOOP CONTROLLED PRESS AND OEDOMETER}

In order to emulate in laboratory the natural soil consolidation process, it is required to impose a vertical compression force on a cylindrical soil sample, which is laterally constrained, under specific conditions. The first condition is that the base of the sample. on which the force is applied, is big in comparison to its height. This assures that the motion of the interstitial fluid is essentially one-dimensional, realising the so-called oedometric condition. The second one is that the pressure increase of the interstitial water that during the compression process can slowly drain through one of the sample's basis remains below suitable low values.

Consolidation tests in which the above conditions are fulfilled are generally of two kind: the so-called IL (Incremental Load) tests and the so-called CRS (Constant Rate of Strain) tests. In It tests the compression force is incremented step by step, according to a geometric progression, at largely spaced fixed time instants. Typically, starting with a value of pressure equal to, say, $25 \mathrm{kPa}$, one doubles it every 24 hours and measures the corresponding axial deformation of the soil sample. By letting the compression force act for 24 lours before increasing it. one guarantees, up to a certain extent, that the pressure of the interstitial water has enough time to stabilise and that it keeps small for most part of the test. However, the evolution of such pressure is not monitored during the test.

In CRS tests ([6], [5]) the soil sample is compressed between two plates, one of which is connected to the piston of a press and the other to a dynamometer. A continuos increasing of the pressure on the soil sample is obtained by moving the piston at a constant speed. The deformation of the soil sample, the value of the average compression stresses and the interstitial pressure of the inner fluid at its base are measured at fixed time instants. By choosing a suitable low speed for the piston, the interstitial pressure at the base of the soil sample stabilises. as desired. at some low value. The piston's speed represents. therefore. the control variable in the consolidation test and the choice of a suitable value corresponds to implementing an open-loop control strategy on the interstitial pressurc. Adequate values of the speed depend on various characteristics of the soil sample. However, keeping the speed very small. one increases the chances of obtaining low values for the interstitial pressure. At the same time. if the speed is small, the value of the compression stress on the soil sample increases linearly. after a transient. as the piston moves. The behaviour of these two variable for a small piston's spced is represented in Figure 1. where $\sigma$ denotes the average compression stress and $\mathrm{C}_{\mathrm{b}}$ denoles the interstitial pressure at the base.

By choosing a higher speed, a possible behaviour of the compression stress $\sigma$ and of the interstitial pressure $U_{b}$ at the base of the soil sample is clescribed in Figure 2. In that situation the test does not meet the required conditions for consolidation testing. Therefore, from a geoteclunical point of vicw, it does not provide useful information on the behaviour of the soil.

If the right conditions are achieved, the measured values of $\sigma$ and $\mathbf{U}_{\mathbf{b}}$ are used for deriving the interstitial pressure $\boldsymbol{u}$ and the effective stress $\sigma^{\prime}=\sigma$ u which is exerted on the solid skeleton of the soil sample. The values of the deformation $\delta$ of the soil samplc, together with measures of water content and of its weight at the beginning and at the end of the lest, are used to derive the Void Index E, which describe the density of the sample. Then, the relation between $\sigma^{\prime}$ and $\mathbf{E}$ is displayed as the curve obtained by linear interpolation of consecutive data in a semilogarithmic representation. The qualitative and quantitative characteristics of such curve. called the Consolidation Curve of the sample, allow one to predict the soil's behaviour under various conditions. A typical Consolidation Curve is that represented in Figure 3.

An evident drawback of both IL tests and CRS tests is their long duration, due to the necessity of letting the load act for a long time or to that of choosing a sinall piston's speed so to guarantee a good behaviour of the interstitial pressure.

To get better performances in terms of time, the equipment for classical CRS tests is modified in such a way that the speed of the piston is no longer constant, but it is determined by a closed-loop control strategy feeding back the actual value of the interstitial pressure ai the sample base. Before describing the way in which such feedback control architecture has been implemented on a suitable hardware, it is necessary to discuss the design guidelines it is based on. 
2.1 Closed-loup control archirecure for soil consolidanion tests

A first step in the synthesis of closed-loop control strategy of the kind we are speaking of consists in choosing a desired behaviour for the controlled variable $\mathbf{U}_{\mathbf{b}}$

A simple choice is that of fixing a desired constant value for $\mathrm{L}_{\mathrm{b}}$. equating it to a suitable percentage of the maximum pressure Pr that can be cierted. using a giren equipment for CRS test. on the soil sample. For remoulded soft soils $\mathcal{C}_{\mathrm{b}}$ should not exceed. in CRS tests. $5 \%$ of the maximum pressure P. While for natural soil it may arrive at 10\% of $\mathrm{Pr}$ (see [6]).

A schematic representation of the control architecture employed in carrying on the consolidation test is. in this first case, the following one:

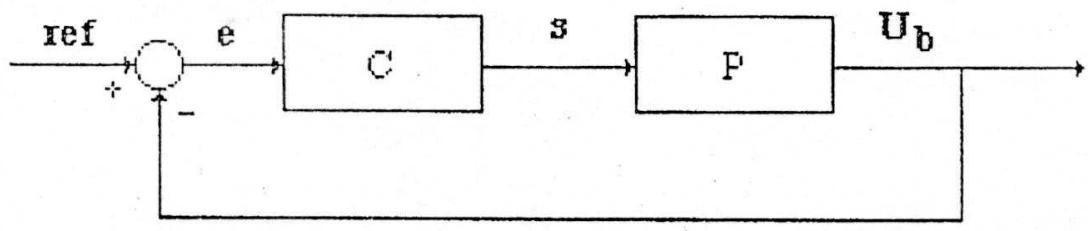

where $P$ represents the laboratory equipment consisting of the oedometer and the press used for testing the soil sample: $\mathrm{C}$ represents the control unit which generates the command $s$, namely the piston's speed. on the base of the difference e (regulation error) between the actual value of the interstitial pressure at the base $\mathbf{U}_{\mathrm{b}}$ and a fixed reference value ref. From a geotechnical point of view, the validity' of a consolidation test carried on in the way described above is assured by the theory underlying CRS tests. In facts, the basic conditions realised in CRS tests are maintained if $\mathrm{U}_{\mathrm{b}}$ is kept equal to a small reference value by a closed-loop strategy. The main advantage, however, is that the piston's speed is increased as much as possible, while keeping $\mathbf{U}_{b}$ constant. This allows to reduce considerably the duration of the test by actually optimising it with respect to the chosen reference value of $\mathrm{U}_{\mathrm{b}}$. In addition, the above control architecture avoids the necessity of fixing an arbitrary piston's speed. as it happens in classical CRS tests. eliminating the risk of inadequatc choices, that can cause the intcrstitial pressure to grow too much, and so reducing the competence the operator is required to have.

A possible alternatively choice. instead of maintaining $\mathbf{U}_{\mathbf{b}}$ constant, is that of keeping constant the ratio $\mathrm{U}_{\mathrm{b}} / \sigma$, while letting the value of $\mathrm{U}_{\mathrm{b}}$ grow as hic compression stress $\sigma$ does. In this sccond case, a schematic representation of the control architecture employed in carrying on the consolidation test is the following :

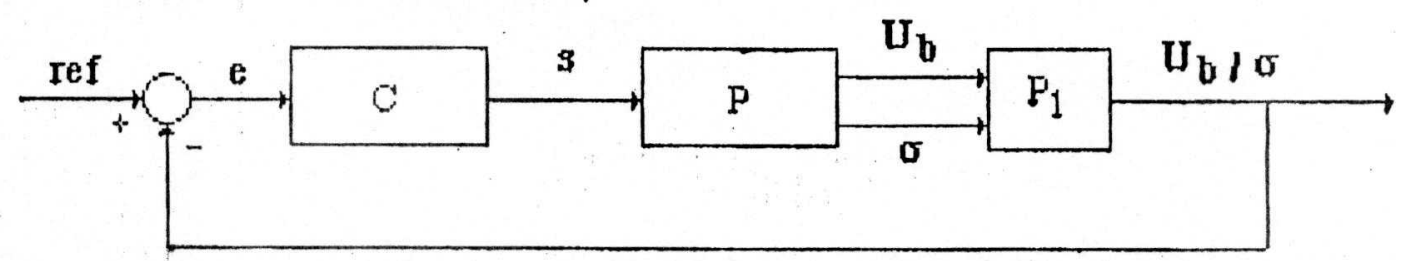

The signals derived from the plant $P$ are the interstitial pressure (measured at the sample's base) $U_{b}$ and the compression stress $\sigma$ applied to the soil sample. The block $P_{1}$ represents a software unit which evaluates the ratio $U_{b} / \sigma$ to be fed back and to be compared with a fixed reference value ref. The regulation error $\mathrm{e}=$ ref $-\mathrm{U}_{\mathrm{b}} / \sigma$ is the variable used by the controller $\mathrm{C}$ to synthesise the command $\mathbf{s}$, representing the piston's speed.

Consolidation tests conducted in this way differ from classical CRS tests, in which the interstitial pressure $U_{b}$ stabilises at some constant value, and cannot rely on the same theoretical framework. However, as we will describe in Section 3, they appear capable to provide data for computing a Consolidation Curve which agrees, with an approximation acceptable for geotechnical applications, with that obtained from classical tests. The advantage in employing the feedback control architecture described above consists in a dramatic reduction (up to several timcs) of test duration (see Section 3). In addition, since no limiting value for $\mathbf{U}_{\mathbf{b}}$ and $\sigma$ are imposed, the flexibility of the test and its capability of producing valuable result for a large variety of soil characteristics are greater than those found in classical CRS tests.

The expcriments made so far indicate that consolidation data obtained using this new kind of test are satisfactorily reliable in comparison with those obtained by means of classical CRS tests (see [3], [4]).

\subsection{Harchiare and software characteristics}

Let us concentrate, in this Section, on the hardware 
and software architecture employed for carrying on consolidation tests by regulating $\mathrm{U}_{\mathrm{b}} / \sigma$ to a constant value. The situation concerning consolidation tests by regulating only $\mathrm{U}_{\mathrm{b}} 10$ a constant value is simpler and. being easily derivable from the other one. will not be described in details.

As mentioned above. the equipment on which the feed-back control architecture has been implemented consists in an oedometric cell with load and pressure transducer. a press and a PC. used as control unit. equipped with an A/D-D/A board.

The piston of the press is moved. through a reduction box. by a SLO-SYN type slepping motor. The mechanical configuration of the reduction box is such that for each rotation of the motor axis the piston moves $0.0533 \mathrm{~mm}$. In order to comply with the characteristics of the chosen A/D-D/A board and of the motor driver and to get an acceptable piston's speed. both during the test and the preliminary positioning of the sample, each motor step has been divided into 20 micro steps. so to split each rotation into 4000 micro steps.

The oedometric cell is equipped with two sensors for mcasuring. respectively. the compression stress $\sigma$ exerted on the soil sample and the interstitial pressure at the base $\mathrm{U}_{\mathrm{b}}$. The measured data are acquired through the A/D-D/A board by the PC, which computes the ratio $\mathrm{U}_{\mathrm{b}} / \sigma$ (block $\mathrm{P}_{1}$ in the above scheme) and the regulation error $\mathbf{e}$.

The control signal s, which represents the piston's speed, is synthesised by means of a PID controller technique (see [1]). More preciscly $\mathbf{s}=\mathbf{s}(\mathbf{t})$ is obtained as sum of an integral term, a proportional term and a derivative term by the following relation

$$
s(t)=T_{i} \int_{0}^{t} e(\tau) d \tau+K e(t)+T_{d} \frac{d e(t)}{d t}
$$

Since the consolidation process depends on the mechanical characteristics of the soil at issue, suitable values of the controller parameters $\mathbf{T}_{\mathbf{i}}, \mathbf{K}, \mathbf{T}_{\mathbf{d}}$ need to be computed for every kind of soil. To explain how this is done. one has to describe some dynamical aspects of the compression process in connection with classical design techniques (see [1]). The classical Ziegler-Nichols Frequency Response Methods for computing appropriate values of the controller parameters requires to apply a proportional control of the form

$$
s(t)=K e(t)
$$

and to increase the gain $\mathrm{K}$ until the process start to oscillate. The controller parameters are then computed using the ultimate value of the gain and the period of oscillation. Actually, due to hardware limitations, the practical tuning of $\mathbf{K}$ turns out to be difficult. In facts, increasing $\mathbf{K}$ the maximum load that is allowed by the dynamometer $(900 \mathrm{Kg}$ in our case) is soon reached. Moreover, if, due to high piston's speed, instability occurs. it is not possible to restore stability by slowing down the piston. Hence. Ziegler-Nichols Frequency Response Method can not be applied.

On the other hand. applying a unit step input signal. the resulting output $\mathrm{U}_{\mathrm{i}} / \sigma$ as an impulsive behariour as described in Figure 4 . This prevents a direct use of Ziegler-Nichols Step Response Melhod. However. by adding a fictitious integrator in front of the process. one get, in response to a step input. an output of the form described in Figure 5 . It is therefore possible to derive the parameters' values for designing a PD controller for the cascade composition of the (fictitious) integrator and the compression process of the form

$$
\bar{s}(t)=T_{i} e(t)+K \frac{d e(t)}{d t} .
$$

Including the fictitious integrator in the controller, the control input to the process turns out to be the integral of $\overline{\mathbf{s}}(\mathbf{t})$, which has an expression given by the first two terms in the second member of (1). By choosing $\mathbf{T}_{\mathbf{d}}=0.25 \mathrm{~T}_{\mathbf{i}}$, as prescribed by ZieglerNichols empirical rules. all the parameters required to implement (1) are computed.

The PID controller so obtained may not work properly during the initial phase of the compression process, when small measured values of $\mathbf{U}_{\mathbf{b}}$ and $\sigma$ are strongly influenced by sensor noise. This may cause the piston's speed and, as a consequence, the interstitial pressure to grow excessively; making impossible to reach the desired value of $\mathbf{U}_{\mathrm{b}} / \sigma$. To prevent this, the controller structure is modified in such a way that its action produces a signal $s(t)$ of the form

$$
s(t)=s_{0} t
$$

linearly increasing the piston's speed, until the normal stress $\sigma$ reaches a prefixed value $\sigma 0$.

In addition, saturation bounds $s_{\min }$ and $s_{\text {max }}$ are imposed in such a way to limit the control action and to avoid very large as well as very small values of $\mathbf{s}$. Globally, the controller has therefore a hybrid structure and its action shifts from (3) to (1) as the compression process develops.

From a geotechnical point of view, the preliminary action of the controller corresponds to a preconsolidation action, which may be necessary for restoring the original stress state of the sample.

The PC that implements the closed-loop control law intcracts with a human operator through a friendly interface. Using the interface commands, the operator can chose the amplitude of the step input used to compute at a preliminary stage the values of the controller parameters, namely for tuning the controller, or he can impose pre-computed values. In 
addition. he can chose the reference value for $\mathrm{U}_{\mathrm{b}} / \sigma$ the parameters $s_{0} . \sigma_{0}$ which determine the switching control action. the sampling time and the saturation bounds $s_{\min }$ and $s_{\max }$.

\section{EXPERIMENTAL RESULTS}

Experimental results depend obviously on the accuracy by which appropriate values of the controller parameters are computed. This requires a preliminary tuning on soil samples of the same kind of that used in the compression test.

Hcre. we report the results obtained on a sample of remoulded material using for the controller parameters the following values:

$K=0.25 ; \mathbf{T}_{\mathbf{i}}=2 \mathrm{~s}: \mathbf{T}_{\mathbf{l}}=0.5 \mathrm{~s}$.

The sampling time has been chosen cqual to $30 \mathrm{~s}$, which is compatible with both the hardware capability and the process dynamics.

The chosen values for the remaining parameters are the following:

$s_{\min }=0.001 \mathrm{~mm} / \mathrm{min}$ :

$\mathrm{s}_{\max }=0.05 \mathrm{~mm} / \mathrm{min}$;

$\mathbf{s}_{0}=0.00003 \mathrm{~mm} / \mathrm{min}^{2} ; \sigma_{0}=50 \mathrm{kPa}$.

The reference value is $\mathrm{U}_{\mathrm{b}} / \sigma=0.05$.

The test consists of a loading phase and an unloading phase, during which data for deducing the related Compression Curve are collected. The total duration of the test has been of 47 hours, about 21 hours for the loading phase and about 26 for the unloading one. The behaviour of $\mathrm{U}_{\mathbf{b}} / \sigma$ during the test is shown in Figure 6. The controller works satisfactorily, achieving the control goal after a relatively short transient. The piston's speed is shown in Figure 7. It increases linearly during pre-compression and then it kecps between the saturation bounds. The drastic variation at about time 1300 corresponds to the phase change between loading and unloading.

A classic CRS test has been performed on a sample of the same material in order to compare the experimental results. The Consolidation Curves obtained by both methods are shown in Figure 8. The thinner line represents the curve obtained by the CRS test and linear interpolation of manually collected data. The other line represents the curve obtained by controlling $\mathbf{U}_{\mathrm{b}} / \sigma$ to the desired value. In bothl cases, the two branches of the curves correspond respectively to the loading and to the unloading phase. The test conducted under closed-loop control provides a large number of data, recorded by the PC, so that, at the size of the Figure, an apparently continuous curve can be directly drawn.

Comparison between the two curves shows a substantial agreement, from a geotechnical point of view, between the results of the two test and this indicates the possibility of substituting both IL and CRS tests in determining the Consolidation Curve.

While the duration of the lest conducted under closed-loop control has been, as said. of 47 hours, for collecting the analogous consolidation data by means of a CRS lest it took about 340 hours. half for the loading phase and half for the unloading one. Similar results have been obtained in other tests of the same kind (see [3]. [4]). indicating that the time required for evaluating the Consolidation Curve can be reduced by about six times.

\section{CONCLUSIONS}

A laboratory cquipment endowed with an automatic closed-loop control for soil consolidation test has been described. The use of automatic closed-loop control allows reducing considerably the duration of the test. providing at the same time data that appear reliable and accurate in comparison with other classical tests.

\section{REFERENCES}

[1] Astrom. K. J. and T. Hagglund -PID controllers: theory, design and tuning- ISA. U.S.A. (1995)

[2] Menzies, B. K. and P. Hooker - PC and local microprocessor controlled geotechnical testing systems - Proc. Int. Conf. Geotechnics and Computer, Paris, (1992)

[3] Mignozzetti. S. - Doctoral disscrtation Universita' di Ancona (1997)

[H] Tenti. F. - Doctoral dissertation - Universita' di Ancona (1997)

[5] Viggiani, C. - Contributo allo studio della consolidazione monodimensionale delle argille Rivișta Ltaliani di Geotecnica, anno 6, n. 4 (1972)

[6] Wissa, A. E., J. T. Christian, E. H. Davis and S. Heiberg - Consolidation at a constant rate of strain J. Soil Mech. Found. Proc. ASCE, vol 97 (1971)

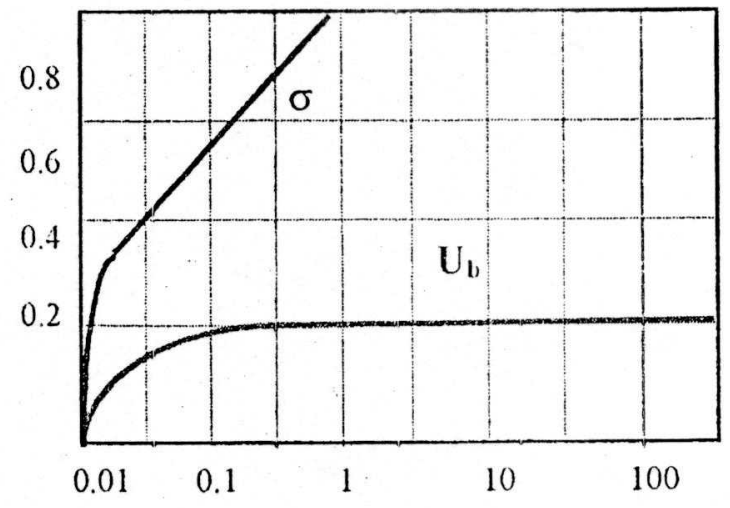

Figure 1. Graphs of $\sigma$ and $\mathbf{U}_{b}$ for low piston's speed. 


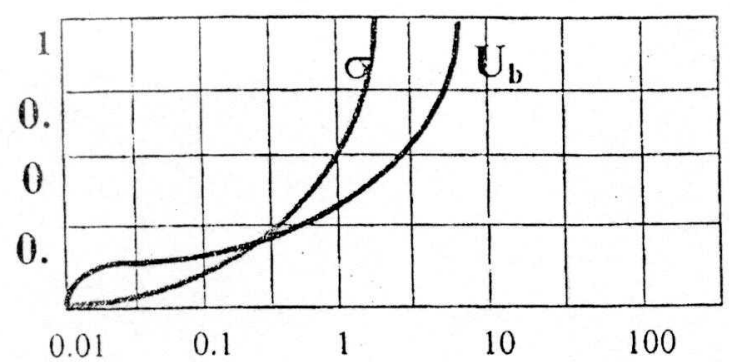

Figure 2. Graphs of $\sigma$ and $\mathbf{U}_{\mathrm{b}}$ for high piston's speed.

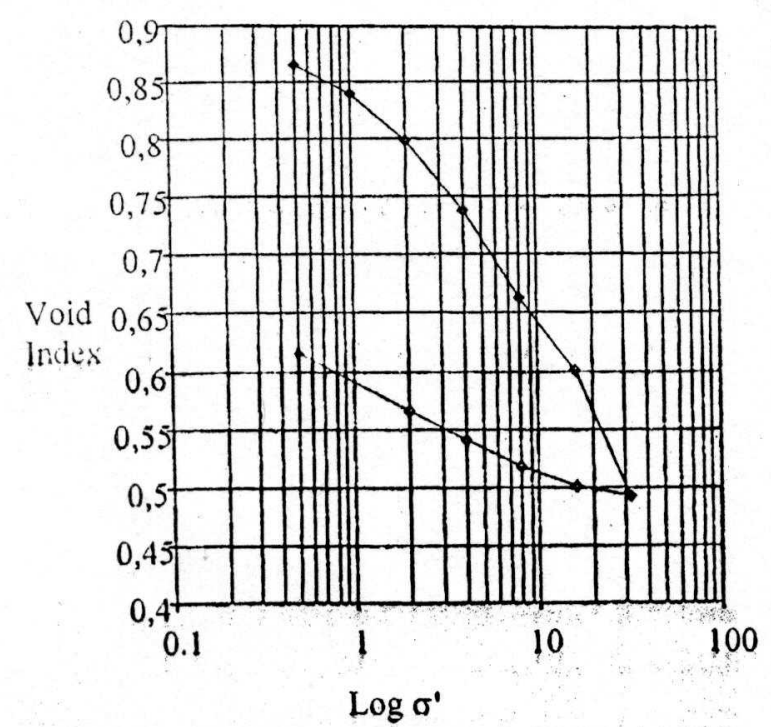

Figure 3. Graph of a typical consolidation curve.

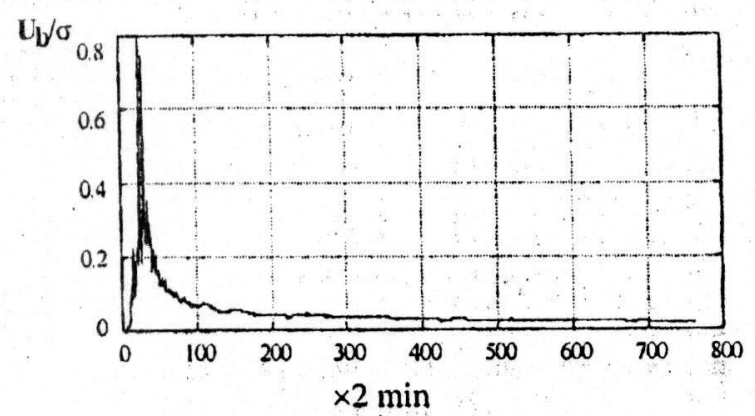

Figure 4. $\mathbf{U}_{\mathrm{b}} / \sigma$ response to a step input.

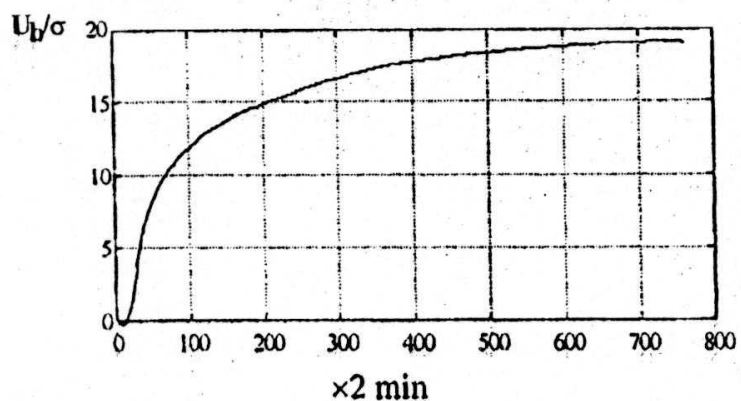

Figure $5 . U_{b} / \sigma$ response with fictitious integrator.

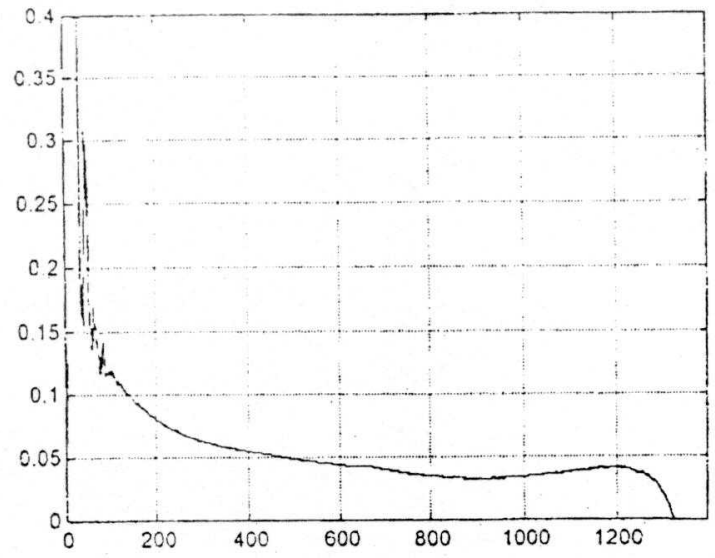

Figure $6 . \mathrm{U}_{\mathbf{b}} / 0$ behaviour during the test.

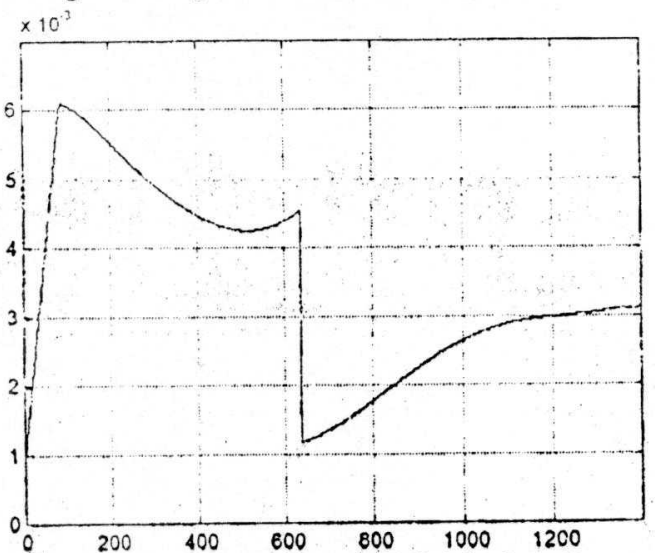

Figure 7. Piston's speed ( $\mathrm{mm} / \mathrm{Min}$ )during the test.

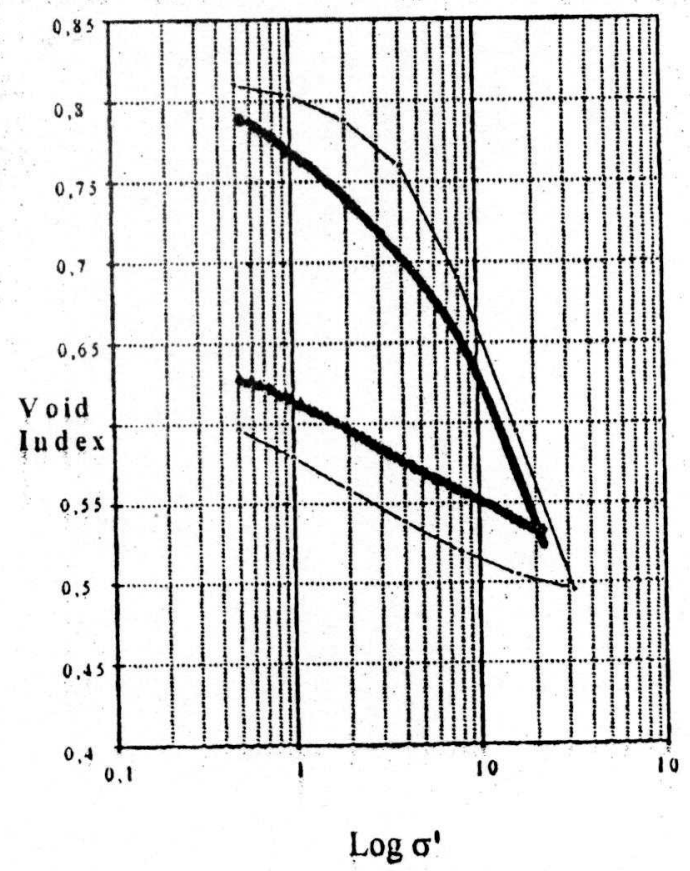

Figure 8. Consolidation curves obtained respectively by CRS test (thinner) and by controlling $\mathbf{U}_{\mathbf{b}} / \sigma$ 